\title{
Influence of spin-orbit interaction within the insulating barrier on the electron transport in Magnetic Tunnel Junctions
}

\author{
A. Vedyayev,,${ }^{1,2, *}$ N. Ryzhanova, ${ }^{1,2}$ N. Strelkov, ${ }^{1,2}$ M. Titova, ${ }^{1}$ M. Chshiev, ${ }^{2}$ \\ B. Rodmacq, ${ }^{2}$ S. Auffret, ${ }^{2}$ L. Cuchet, ${ }^{2}$ L. Nistor, ${ }^{3}$ and B. Dieny ${ }^{2}, \dagger$ \\ ${ }^{1}$ Department of Physics, Moscow Lomonosov State University, Moscow 119991, Russia \\ ${ }^{2}$ SPINTEC, Univ. Grenoble Alpes / CEA / CNRS, 38000 Grenoble, France \\ ${ }^{3}$ Applied Materials, 38000 Grenoble, France
}

\begin{abstract}
We present a theory of the Anisotropy of Tunneling Magnetoresistance (ATMR) phenomenon in magnetic tunnel junctions (MTJ) attributed to Rashba spin-orbit interaction in the insulating barrier. ATMR represents the difference of tunnel magnetoresistance (TMR) amplitude measured with in-plane and out-of-plane magnetic configurations. It is demonstrated that within the spinpolarized free electron model, the change of conductance associated with the ATMR is exactly twice of the change of conductance measured at full saturation (i.e. in parallel configuration of magnetizations) between in-plane and out-of-plane configuration, i.e. the tunneling anisotropic magnetoresistance (TAMR). Both ATMR and TAMR are closely related to the TMR amplitude and spin-orbit constant. The predicted ATMR phenomenon is confirmed experimentally, showing a few percent values in case of the widely studied $\mathrm{CoFeB} / \mathrm{MgO} / \mathrm{CoFeB}$ based MTJ.
\end{abstract}

Keywords: Spintronics, Spin Hall effect, Rashba effect, SOI, ATMR, TAMR

Spin-orbit interactions (SOI) are at the origin of several transport properties of bulk ferromagnetic metals, such as Anomalous Hall Effect (AHE) [1, 2] and Anisotropic Magnetoresistance (AMR) [3]. More recently a lot of attention was paid to the influence of spinorbit coupling (Rashba [4] or Spin Hall effect [5]) on the non-equilibrium spin-orbit torque in single nanomagnet [4] and on the Tunneling Anisotropic Magnetoresistance (TAMR) of Magnetic Tunnel Junctions (MTJ) [69]. The TAMR phenomenon in MTJ is usually measured at full saturation of the MTJ and consists in a variation of the tunnel resistance in parallel magnetic configuration as a function of the direction of the magnetization with respect to the crystallographic axis. It is measured either under a rotating saturation field in tunnel junctions with two magnetic electrodes [9] or in junctions with only one ferromagnetic [10-12] or antiferromagnetic [13] electrode. The TAMR is of different origin compared to TMR [14]. One origin of TAMR $[6,7,10,15]$ is the Rashba interaction which arises from the gradient of electrical potential at the interfaces between the ferromagnetic layer and nonmagnetic heavy metal, semiconductor or insulator layer. It was shown that this type of SOI influences the interfacial density of states (DOS) in the ferromagnetic layer resulting in a dependence of the DOS on the direction of the magnetization vector with respect to the crystallographic axes. As a result, the tunneling current depends on the angle between the magnetization and crystallographic axes.

In this paper, we present a theoretical and experimental study of the anisotropy of the TMR (ATMR) in magnetic tunnel junctions in presence of Rashba SOI within the tunnel barrier. The ATMR in MTJs differs from the TAMR as follows. Let us define $R_{x}$, $R_{x \mathrm{AP}}\left(R_{z \mathrm{P}}, R_{z \mathrm{AP}}\right)$ the MTJ resistances in parallel (P) and antiparallel (AP) configuration with the magnetization of the two electrodes being in-plane (x-direction) (out-of-plane (z-direction)). The TAMR is defined as $\mathrm{TAMR}=\mathrm{TAMR}_{\mathrm{P}}=\left(R_{z \mathrm{P}}-R_{x \mathrm{P}}\right) / R_{x} \mathrm{P}$. It is usually measured in parallel magnetic configuration. It can also be defined as $\mathrm{TAMR}_{\mathrm{AP}}$ in antiparallel configuration, i.e. $\mathrm{TAMR}_{\mathrm{AP}}=\left(R_{z \mathrm{AP}}-R_{x} \mathrm{AP}\right) / R_{x} \mathrm{AP}$. In contrast, in this work, we introduce the ATMR defined as:

$$
\mathrm{ATMR}=\frac{R_{x \mathrm{AP}}-R_{x \mathrm{P}}}{R_{x \mathrm{P}}}-\frac{R_{z \mathrm{AP}}-R_{z \mathrm{P}}}{R_{z \mathrm{P}}} .
$$

Considering that the TAMR $_{P}$ is weak [9], ATMR $\mathrm{TAMR}_{\mathrm{P}}-\mathrm{TAMR}_{\mathrm{AP}}$. The ATMR represents the variation of the TMR amplitude between in-plane magnetic configuration and out-of-plane magnetic configuration due to SOI. It is interesting to note that such dependence of the magnetoresistance amplitude on the direction of the current with respect to the magnetization has already been observed with the giant magnetoresistance in spin-valves [16]. In MTJs, from a general experimental point of view, the TMR of $\mathrm{MgO}$ based in-plane magnetized MTJs has reached values above $600 \%$ [17, 18]. In contrast, the largest TMR of $\mathrm{MgO}$ based out-of-plane magnetized MTJs is in the range (200-350\% [19]), significantly lower than the values obtained in their in-plane magnetized counterparts. In theory, the TMR amplitude predicted by ab-initio calculations neglecting spinorbit [14] is much larger than the experimentally obtained values. In experiments, the TMR amplitude is limited by defects which can have several origins: interdiffusion of metallic species in the composite magnetic electrodes [17, 18, 20], structural defects associated with fcc/bcc in-stack structural competition [21], presence of dislocations in $\mathrm{MgO}$ associated with $\mathrm{Fe}(\mathrm{Co})-\mathrm{MgO}$ crystallographic mismatch, adsorbed water molecules etc. 
However, considering the significant difference of TMR observed between in-plane and out-of-plane magnetized MTJs, it is interesting to investigate whether an intrinsic origin of this difference can be attributed to spin-orbit effects. In this study, we developed an analytic description of ATMR in free electron model as it was carried out in [22] for TMR and show that correlations exist between ATMR, TAMR and TMR. Furthermore, we confirm our theoretical predictions by experimental investigation of the ATMR behavior in MgO based MTJs with orthogonal anisotropies, i.e. with one electrode being magnetized in-plane and the other being magnetized out-of-plane. By applying a saturation field successively in-plane then outof-plane, we could derive the ATMR amplitude in these $\mathrm{MgO}$ based MTJs. Its amplitude is quite weak which is consistent with earlier TAMR measurements on similar MTJs [9] and the TAMR AP is found to be opposite to the TAMR $_{P}$.

In MTJs, spin-orbit coupling appears within the barrier due to the gradient of voltage across the barrier (Rashba effect) or due to the presence of impurities of heavy ions embedded into the barrier. In the $\mathrm{MgO}$ based MTJs of practical interest in particular for Magnetic Random Access Memories, the amount of heavy metal impurities in the tunnel barrier is negligible so that the dominant source of spin-orbit coupling is in this case the Rashba effect.

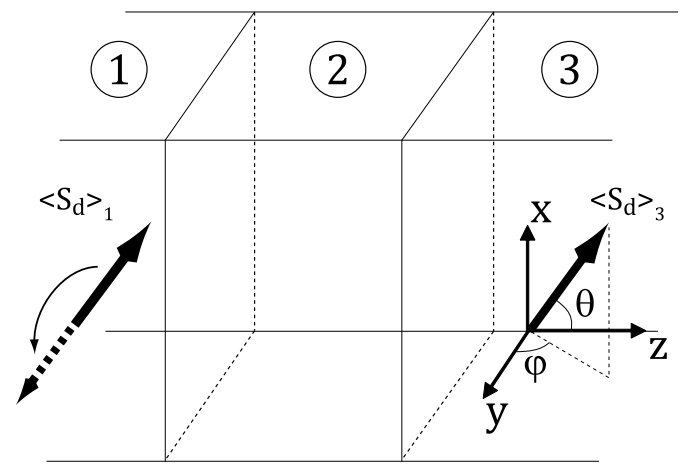

FIG. 1: Sandwitch structure. 1,3-ferromagnetic layers, 2 insulator. Averaged spin $\left\langle\hat{S}_{d}\right\rangle_{1}$ is collinear (P or AP) to the $<\hat{S}_{d}>_{3}$.

Earlier, the AHE in MTJs due to the spin-orbit scattering in the barrier [23] or Rashba type spin-orbit coupling [24] were investigated. Here below, the influence of spin-orbit coupling due to the gradient of voltage within the barrier (Rashba effect) on the tunnel magnetoresistance of MTJ and on the anisotropy of this TMR is theoretically addressed. Let us describe the MTJ as a magnetic sandwich consisting of two ferromagnetic metals separated by an insulating barrier. The Hamiltonian of the system can be written for the free electron model in mean field approximation as follows:

$$
\hat{H}=\frac{\hat{p}^{2}}{2 m}-J_{s d} \hat{\sigma}\left\langle\hat{S}_{d}\right\rangle
$$

for F-layer, where $J_{s d}$ is the exchange interaction of the itinerant $s$-electrons with the localized $d$-electrons, $\hat{\sigma}-$ Pauli matrix and $<\hat{S}_{d}>$ is the averaged value of $d$ electron spin described as a unit vector in the direction of the local magnetization:

$$
\left\langle\hat{S}_{d}\right\rangle=(\sin \theta \cos \varphi ; \sin \theta \sin \varphi ; \cos \theta)
$$

In the barrier, the Hamiltonian is written:

$$
\langle\hat{H}\rangle=\frac{\hat{p}^{2}}{2 m}+U+i \alpha\left(\sigma\left[\vec{z} \times \frac{\partial}{\partial \vec{r}}\right]\right),
$$

where $\alpha=\lambda^{2} \mathrm{eV} / d, \lambda$ being the effective Compton length, $d$ - is the barrier thickness, $U$ its height, $V$ - drop of applied voltage across the barrier, $\vec{z}$ is unit vector in $z$ direction (see Fig.1). The solutions of Schrödinger equation with Hamiltonian (2)-(4) for the wave functions in all three layers for $\theta=\pi / 2$ or $\theta=0$ and $\varphi=0$ can be easily derived.

We now calculate the densities of currents $J$ at first order on voltage for both directions of spin projections substituting the calculated expressions for the wave functions into the expression:

$$
\begin{aligned}
J^{\uparrow(\downarrow)} & =-\frac{e^{2}}{2 \pi \hbar} \frac{1}{(2 \pi)^{2}} \int J_{\varkappa, \varepsilon, \gamma}^{\uparrow(\downarrow)} \frac{\partial f}{\partial \varepsilon} d \gamma d \varepsilon \varkappa d \varkappa, \\
J_{\varkappa, \varepsilon, \gamma}^{\uparrow(\downarrow)} & =\operatorname{Im} \psi^{*} \frac{\partial \psi}{\partial z}
\end{aligned}
$$

where $J_{\varkappa, \varepsilon, \gamma}^{\uparrow(\downarrow)}$ is the dimensionless partial current density for spin up and down projections and for given values $\varkappa$, $\varepsilon$ and angle $\gamma$.

We are interested in the value of the tunneling anisotropic magnetoresistance (TAMR) and anisotropy of tunnel magnetoresistance (ATMR). In the limit of thick barrier, we have:

$$
\begin{aligned}
& \Delta J_{\mathrm{TAMR}}^{\mathrm{P}(\mathrm{AP})}=\left.J_{\varkappa, \varepsilon}\right|_{\theta=\frac{\pi}{2}, \varphi=0}-\left.J_{\varkappa, \varepsilon}\right|_{\theta=0, \varphi=0}= \\
& \pm(\Delta E)^{2} \frac{8 q_{0}^{2} \mathrm{e}^{-2 q_{0} d}\left(q_{0}^{2}-k_{1} k_{2}\right)^{2}}{\left(q_{0}^{2}+k_{1}^{2}\right)^{2}\left(q_{0}^{2}+k_{2}^{2}\right)^{2}} \times \\
& \left(k_{1}-k_{2}\right)^{2}= \pm(\Delta E)^{2} \frac{1}{2} \mathrm{TMR}, \\
& \Delta E=\left.E_{+}\right|_{z=z_{2}}-\left.E_{-}\right|_{z=z_{2}} \approx \frac{2 m}{\hbar^{2}} \frac{d}{q_{0}} \alpha|\varkappa|
\end{aligned}
$$

where TMR is the amplitude of the tunnel magnetoresistance expressed as $\left(J^{\mathrm{P}}-J^{\mathrm{AP}}\right)$ for a given voltage, without 
spin-orbit contributions,

$$
\begin{aligned}
E_{ \pm}(z) & =\mathrm{e}^{q_{ \pm}\left(z-z_{1}\right)}, \\
q_{ \pm} & =\sqrt{q_{0}^{2} \pm \frac{2 m}{\hbar^{2}} \alpha|\varkappa|}, \\
q_{0}^{2} & =\frac{2 m}{\hbar^{2}}\left(U-E_{F}+\varepsilon\right)+\varkappa^{2}, \\
k_{1(2)} & =\sqrt{k_{F}^{\uparrow(\downarrow) 2}-\varkappa^{2}-\frac{2 m}{\hbar^{2}}} \varepsilon,
\end{aligned}
$$

where $E_{F}-$ Fermi energy. The difference of $q_{+}-q_{-}$ is due to the splitting of the evanescent bands in the barrier caused by SOI. In (6), it is interesting to note that $\Delta J_{\mathrm{TAMR}}^{\mathrm{P}}$ is exactly opposite to $\Delta J_{\mathrm{TAMR}}^{\mathrm{AP}}$. As a result:

$$
\begin{gathered}
\Delta J_{\mathrm{ATMR}}=\left.\left(J^{\mathrm{P}}-J^{\mathrm{AP}}\right)\right|_{\theta=\frac{\pi}{2}, \varphi=0}- \\
-\left.\left(J^{\mathrm{P}}-J^{\mathrm{AP}}\right)\right|_{\theta=0, \varphi=0}=2 \Delta J_{\mathrm{TAMR}}^{\mathrm{P}}
\end{gathered}
$$

(7) indicates that the TAMR is proportional to TMR before integration over $\varepsilon$ and $\varkappa$, so that the TAMR/TMR ratio gives the value of the spin orbit amplitude $\alpha$. For a given $\alpha$, the larger the TMR, the larger the TAMR. The most dramatic changes in electron transport due

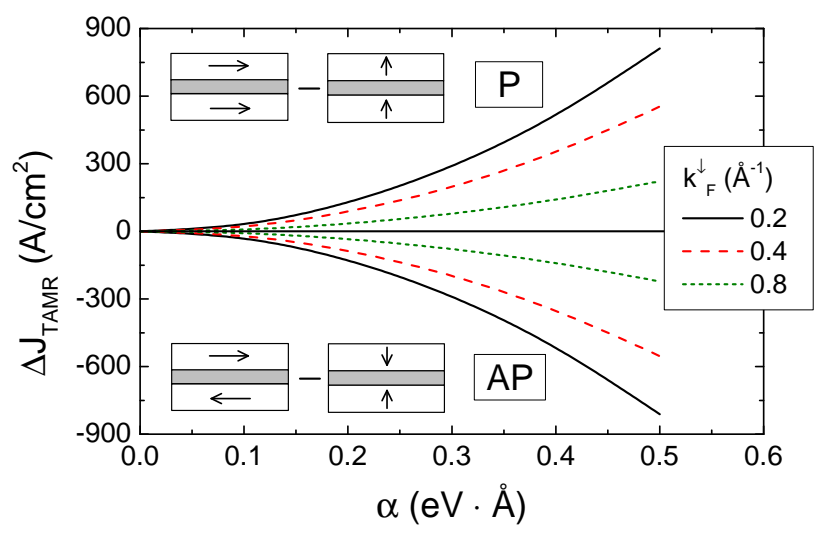

FIG. 2: The dependence of the absolute TAMR $\left(J_{\theta=\pi / 2}-\right.$ $J_{\theta=0}$ ) at a given voltage $V=0.5 \mathrm{~V}$ on the value of Rashba constant $\alpha$. For $\theta=\pi / 2$, the direction of magnetization is perpendicular to the current (in-plane magnetization) and for $\theta=0$ it is parallel to the current (out-of-plane magnetization). Upper half of the figure corresponds to the configuration with parallel directions of magnetizations of F-electrodes (magnetic configurations shown in top inset) and lower half to the antiparallel one (magnetic configurations shown in bottom inset). The curves are given for different values of $k_{F}^{\downarrow}\left(\AA^{-1}\right)$ and fixed $k_{F}^{\uparrow}=1 \AA^{-1}, q_{0}=1 \AA^{-1}, d=7 \AA$. The values of constant $\alpha$ are given in the Table 1 of [4]. They vary in the interval $0.01-1$.

to the influence of spin-orbit interaction is expected in MTJs comprising half-metallic ferromagnetic electrodes. In this case, in the antiparallel magnetic configuration, the density of current without spin-orbit interaction is equal to zero. In presence of SOI, a tunneling current appears which density is proportional to the square of the spin-orbit interaction $(\Delta E)^{2}$. As a result, the tunnel magnetoresistance $\left(J^{P}-J^{A P}\right) / J^{A P}$ remains finite. For weak values of $\alpha$ in the range $0.01-0.1 \mathrm{eV} \cdot \AA$, the TMR should reach very large amplitude in the range $10^{3}-$ $10^{5}$. However, in experiments, TMR amplitude of only $\sim 850 \%$ at low $T$ were observed [25]. This rather low experimental TMR value obtained in half-metal based MTJs can be explained by the presence of significant SOI. Another interesting feature of the influence of spin-orbit interaction on TAMR for the antiparallel configuration of half-metallic electrodes is the expected abnormal high value of anisotropic magnetoresistance in AP configuration: $J^{A P}(\theta=0) / J^{A P}(\theta=\pi / 2)=2$. Fig. 2 shows the variation of the absolute TAMR in the $\mathrm{P}$ and AP configurations versus Rashba constant $\alpha$ for different values of $k_{F}^{\downarrow}$ for fixed $k_{F}^{\uparrow}$. As already pointed out, the signs of TAMR are opposite for the $\mathrm{P}$ and AP configurations. We may notice that the absolute values of TAMR $\left(\Delta J_{\mathrm{TAMR}}\right)$ for $\mathrm{P}$ and $\mathrm{AP}$ configurations are the same, but the absolute relative value of TAMR defined as $\Delta J_{\mathrm{TAMR}} / J_{\theta=0}$ is larger in the AP configuration due to the difference of total currents for $\mathrm{P}$ and $\mathrm{AP}$ configurations.

The TAMR in $\mathrm{MgO}$ and $\mathrm{Al}_{2} \mathrm{O}_{3}$-based MTJ has already been measured in [9] in parallel magnetic configuration by applying a rotating field large enough (7T) to saturate the magnetization of both electrodes along the field direction. A well-defined TAMR signal was observed at $10 \mathrm{~K}$ with however a rather small amplitude in the range $0.1-0.3 \%$. In the present experimental study, the ATMR of MgO-based MTJ was investigated. Measuring the ATMR requires to be able to set the sample in AP and $\mathrm{P}$ magnetic configurations the magnetization of both magnetic electrodes being once oriented in-plane, once oriented out-of-plane. Practically, this is possible in $\mathrm{P}$ configuration by applying a saturation field respectively in-plane and out-of-plane. However, achieving this for the AP configuration would require the sample to exhibit a strong cubic anisotropy with easy axis both out-of-plane and in-plane or an antiferromagnetic coupling through the tunnel barrier larger than the anisotropy energy. None of these requirements are satisfied in sputtered $\mathrm{CoFeB} / \mathrm{MgO}$-based MTJs. We therefore decided to design a sample in which the two magnetic electrodes have orthogonal anisotropies: one has out-ofplane anisotropy, the other easy-plane anisotropy. This can be obtained by carefully adjusting the thickness of each $\mathrm{CoFeB}$ layer so that in one electrode the interfacial $\mathrm{PMA}$ at $\mathrm{CoFeB} / \mathrm{MgO}$ interface dominates the demagnetizing energy of the corresponding CoFeB layer whereas for the other electrode, the opposite is true. Then by performing two sets of magnetoresistance measurements versus field, using the current-in-plane tunneling setup (CIPTMR)[26] one with field applied in-plane, the other with field out-of-plane up to full saturation, one can 
probe on the same sample the TMR amplitude associated with a change in relative orientation of magnetization from $90^{\circ}$ to $0^{\circ}$ with $\mathrm{P}$ final state either in-plane or outof-plane. Here one must make sure that the full satura-

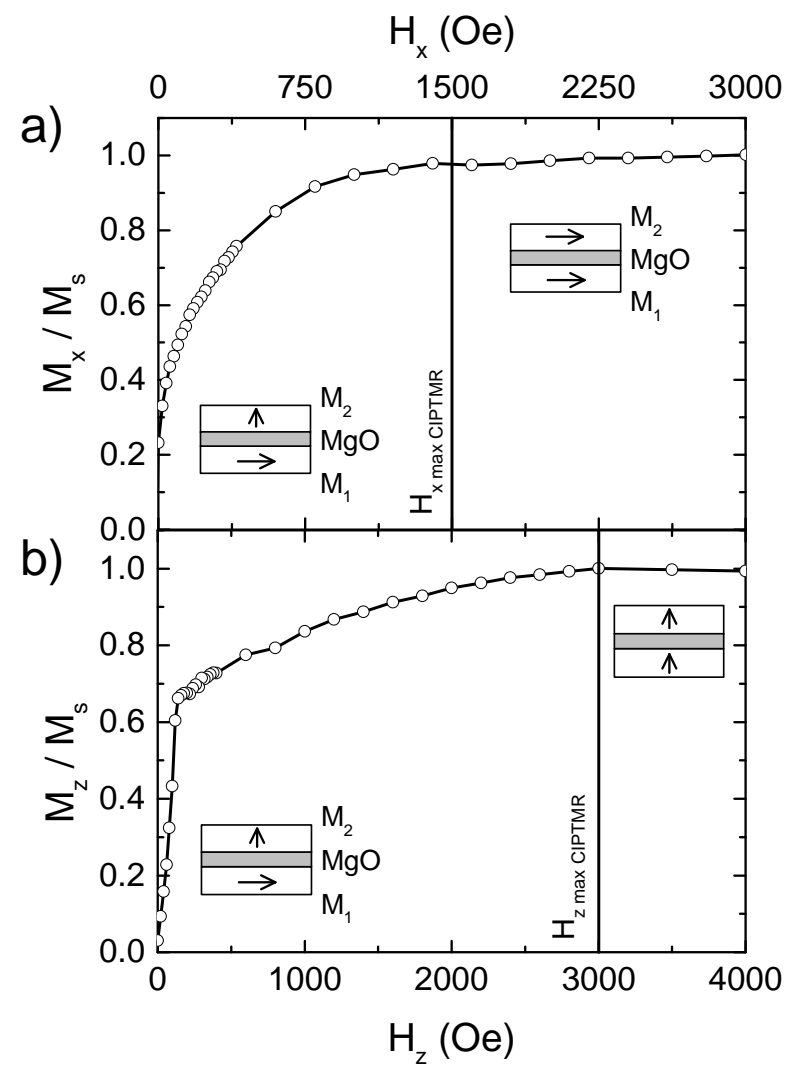

FIG. 3: Normalized magnetization versus field. a) Field applied in-plane (x-direction); (b) Field applied out-of-plane (zdirection). The vertical lines indicate the corresponding maximum field available in the CIPTMR experimental setups.

tion can be reached with the maximum fields available in the CIPTMR measurement setup both with in-plane and out-of-plane field. In our setups, these maximum fields are respectively $H_{x \max \text { CIPTMR }}=1.5 \mathrm{kOe}$ for the in-plane field configuration and $H_{z \max \text { CIPTMR }}=3 \mathrm{kOe}$ for the outof-plane field configuration. As a result, the thicknesses of the two magnetic electrodes $\left(t_{1}\right.$ and $\left.t_{2}\right)$ must be chosen to fulfill the following equations:

$$
\begin{aligned}
& 0<\frac{2}{M_{1 s}}\left(\frac{K_{1 s}}{t_{1}}-2 \pi M_{1 s}^{2}\right)<H_{x \max \text { CIPTMR }} \\
& 0<\frac{2}{M_{2 s}}\left(2 \pi M_{2 s}^{2}-\frac{K_{2 s}}{t_{2}}\right)<H_{z \max \text { CIPTMR }}
\end{aligned}
$$

where $M_{s}, K_{s}$ and $t$ represent respectively the saturation magnetization, interfacial PMA and thickness of the two magnetic eletcrodes. After a detailed study of the influence of the electrode thickness on the magnetic effective anisotropy of bottom and top electrodes [27], the following sample composition was chosen fulfilling the required conditions: $\mathrm{Ta}(3 \mathrm{~nm}) / \mathrm{CoFeB}(1.15 \mathrm{~nm}) / \mathrm{MgO}(1.4 \mathrm{~nm}) /$ $\mathrm{FeCoB}(1.6 \mathrm{~nm}) / \mathrm{Ta}(1 \mathrm{~nm}) / \mathrm{Pt}(2 \mathrm{~nm})$. The MgO barrier was formed by a two step natural oxidation of an $\mathrm{Mg}$ layer under $150 \mathrm{mbar}$ oxygen for $10 \mathrm{~s}$. The samples were subsequently annealed $1 \mathrm{~h}$ at $300^{\circ} \mathrm{C}$. The $1.15 \mathrm{~nm}$ thick bottom electrode which has a weaker interfacial anisotropy (Co rich alloy) has in-plane magnetization (electrode 2) whereas the thicker top electrode which is Fe rich has out-of-plane anisotropy (electrode 1).

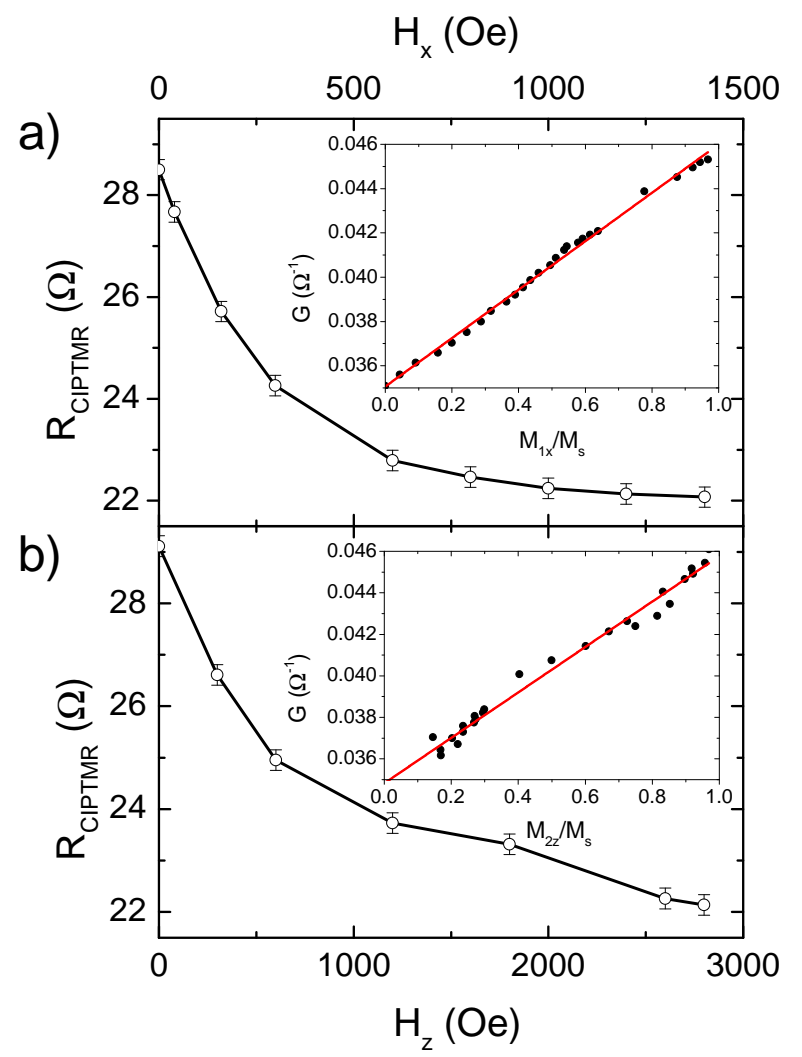

FIG. 4: $R_{\text {CIPTMR }}$ versus field. a) Field applied in-plane (xdirection); (b) Field applied out-of-plane (z-direction). Inset: Conductance (inverse of $R_{\text {CIPTMR }}$ ) versus the reduced component of the rotating magnetization along the applied field direction. The red line represents a linear fit of the experimental variation.

Magnetization measurements were first performed using a vibrating sample magnetometer (VSM) with inplane and out-of-plane field (See Fig. 3). Fig. 3a shows that in-plane saturation of the top electrode magnetization is reached at about $1.3 \mathrm{kOe}$, below the $1.5 \mathrm{kOe}$ maximum field available in the CIPTMR setup with in-plane field configuration. Conversely, Fig. $3 \mathrm{~b}$ indicates that the out-of-plane saturation of the bottom electrode is reached at a field $\sim 3 \mathrm{kOe}$ low enough so that saturation can be reached in the CIPTMR setup with out-of-plane 
field configuration. The rapid rise of magnetization at low fields $(<0.1 \mathrm{kOe})$ seen in Fig. $3 \mathrm{~b}$ is due to the fact that the top electrode at $H_{z}=0$ is in multidomain up and down state and rapidly gets saturated out-of-plane upon $H_{z}$ field application.

Next, CIPTMR measurements were performed with field applied in-plane and out-of-plane up to full saturation (See. Fig. 4). A gradual decrease of the resistance is observed in both cases due to the change in relative orientation between the magnetization of the two electrodes from $90^{\circ}$ to $0^{\circ}$. By combining the data of Fig. 3 and Fig. 4, we plotted in the insets the variation of conductance (inverse of resistance) versus the component of rotating magnetization along the applied field direction.

Assuming that the conductance varies as a linear function of the scalar product between the magnetization of the two electrodes [22], linear fittings of the conductance versus component of the rotating magnetization along field direction were performed forcing the conductance at $H=0$ to be the same in the two cases. The following fitting equations were obtained: $G\left(\Omega^{-1}\right)$ $=\left(0.034984 \pm 8 \cdot 10^{-5}\right)+\left(0.01106 \pm 7 \cdot 10^{-4}\right) M_{1 x} / M_{s}$ for inplane field (Fig. 4a) and $G\left(\Omega^{-1}\right)=\left(0.034984 \pm 8 \cdot 10^{-5}\right)+$ $\left(0.01071 \pm 1.4 \cdot 10^{-4}\right) M_{2 x} / M_{s}$ for out-of-plane field. From these values, by extrapolation, one can derive the full TMR amplitude measured between $\mathrm{P}$ and AP configurations in in-plane and out-of-plane configurations. The following values were obtained: $R_{x \min }=21.72 \pm 0.15 \Omega$, $R_{x \max }=41.80 \pm 0.29 \Omega$ yielding $(\Delta R / R)_{x}=92.4 \% \pm 0.8 \%$ for the in-plane configuration and $R_{z \min }=21.88 \pm 0.29 \Omega$ and $R_{z \max }=41.20 \pm 0.55 \Omega$ for the out-of-plane configuration yielding $(\Delta R / R)_{z}=88.3 \% \pm 2.3 \%$.

It appears clearly from these results that the difference of TMR measured in-plane and out-of-plane is weak. Therefore the large difference in TMR amplitude generally observed between in-plane magnetized MTJs (up to $600 \%$ at RT) and out-of-plane magnetized MTJs (up to $350 \%$ ) is not due to an intrinsic effect related to spin-orbit coupling but likely to a poorer growth quality in out-ofplane magnetized MTJ due to the in-stack coexistence of fcc and bcc layers or to the use of thinner magnetic layers in perpendicular MTJs or to the presence of impurities in or very next to the the tunnel barrier.

More quantitatively, the error bars are unfortunately too large to make definite conclusion but the observed difference between $R_{z \text { min }}$ and $R_{x \text { min }}$ is consistent in amplitude (a few tenths of \%) and sign with the previous measurement of TAMR reported in [9]. Furthermore the TAMR measured in $\mathrm{P}$ and AP configurations $\left(R_{z \text { min }}-R_{x \text { min }}\right.$ versus $\left.R_{z \max }-R_{x \max }\right)$ have opposite sign which is consistent with expression (6) and the calculation shown in Fig. 2. In addition, the weak difference in magnetoresistance amplitude measured in-plane and out-of-plane (92.4\% vs $88.3 \%$ ) represents an ATMR of $4.6 \pm 3.2 \%$ at room temperature. This is the right expected order of magnitude but the uncertainty is too large to reasonably try to extract a corresponding value of the spin orbit constant.

In conclusion, the anisotropy of the TMR (ATMR) was investigated both theoretically and experimentally in $\mathrm{MgO}$-based magnetic tunnel junctions. Theoretically, it was shown that under the model assumption, the ATMR amplitude has twice the TAMR amplitude measured in parallel configuration. This derives from the fact that the TAMR (expressed as absolute variation of current or conductance at given voltage) in $\mathrm{P}$ configuration is just opposite to the TAMR in AP configuration. These effects were semi-quantitatively confirmed by experiments performed on MTJs having orthogonal anisotropies (one electrode having easy-plane anisotropy, the other perpendicularto-plane anisotropy). These spin orbit effects are too weak to explain the large difference in TMR amplitude generally observed between in-plane magnetized MTJs (up to $600 \%$ ) and out-of-plane magnetized MTJs (up to $350 \%)$. This difference is more likely related to differences in stack growth quality and thickness of the magnetic layers.

Acknowledgements: This work was partly funded by ERC Adv grant MAGICAL n ${ }^{\circ} 669204$.

* Electronic address: vedy@magn.ru

Electronic address: bernard.dieny@cea.fr

[1] P. Wölfle and K. A. Muttalib, Phil. Trans. R. Soc. A 15, 508 (2006).

[2] N. Nagaosa, J. Phys. Soc. Jpn. 75, 042001 (2006).

[3] T. R. McGuire and R. I. Potter, IEEE Trans. Magn. 11, 1018 (1975).

[4] P. Gambardella and I. M. Miron, Phil. Trans. R. Soc. A 369, 3175 (2011).

[5] L. Liu, C.-F. Pai, Y. Li, H. W. Tseng, D. C. Ralph, and R. A. Buhrman, Science 336, 555 (2012).

[6] A. N. Chantis, K. D. Belashchenko, E. Y. Tsymbal, and M. van Schilfgaarde, Phys. Rev. Lett. 98, 046601 (2007).

[7] A. B. Shick, F. Máca, J. Mašek, and T. Jungwirth, Phys. Rev. B 73, 024418 (2006).

[8] J. D. Burton and E. Y. Tsymbal, Phys. Rev. B 93, 024419 (2016).

[9] L. Gao, X. Jiang, S.-H. Yang, J. D. Burton, E. Y. Tsymbal, and S. S. P. Parkin, Phys. Rev. Lett. 99, 226602 (2007).

[10] A. Matos-Abiague, M. Gmitra, and J. Fabian, Phys. Rev. B 80, 045312 (2009).

[11] K. Wang, T. L. A. Tran, P. Brinks, J. G. M. Sanderink, T. Bolhuis, W. G. van der Wiel, and M. P. de Jong, Phys. Rev. B 88, 054407 (2013).

[12] K. Wang, J. G. M. Sanderink, T. Bolhuis, W. G. van der Wiel, and M. P. de Jong, Phys. Rev. B 89, 174419 (2014).

[13] Y. Y. Wang, C. Song, B. Cui, G. Y. Wang, F. Zeng, and F. Pan, Phys. Rev. Lett. 109, 137201 (2012).

[14] W. H. Butler, X.-G. Zhang, T. C. Schulthess, and J. M. MacLaren, Phys. Rev. B 63, 054416 (2001).

[15] M. N. Khan, J. Henk, and P. Bruno, Journal of Physics: Condensed Matter 20, 155208 (2008). 
[16] B. Dieny, C. Cowache, A. Nossov, P. Dauguet, J. Chaussy, and P. Gandit, Journal of Applied Physics 79, 6370 (1996).

[17] S. Ikeda, J. Hayakawa, Y. Ashizawa, Y. M. Lee, K. Miura, H. Hasegawa, M. Tsunoda, F. Matsukura, and H. Ohno, Applied Physics Letters 93, 082508 (2008).

[18] S. V. Karthik, Y. K. Takahashi, T. Ohkubo, K. Hono, H. D. Gan, S. Ikeda, and H. Ohno, Journal of Applied Physics 111, 083922 (2012).

[19] M. Krounbi, V. Nikitin, D. Apalkov, J. Lee, X. Tang, R. Beach, D. Erickson, and E. Chen, ECS Transactions 69, 119 (2015)

[20] Y. M. Lee, J. Hayakawa, S. Ikeda, F. Matsukura, and H. Ohno, Applied Physics Letters 89, 042506 (2006).

[21] S. Yuasa and D. D. Djayaprawira, Journal of Physics D:
Applied Physics 40, R337 (2007).

[22] J. C. Slonczewski, Phys. Rev. B 39, 6995 (1989).

[23] A. Vedyayev, N. Ryzhanova, N. Strelkov, and B. Dieny, Phys. Rev. Lett. 110, 247204 (2013).

[24] A. V. Vedyayev, M. S. Titova, N. V. Ryzhanova, M. Y. Zhuravlev, and E. Y. Tsymbal, Appl. Phys. Lett. 103, 032406 (2013)

[25] N. Tezuka, N. Ikeda, F. Mitsuhashi, and S. Sugimoto, Applied Physics Letters 94, 162504 (2009).

[26] D. C. Worledge and P. L. Trouilloud, Applied Physics Letters 83, 84 (2003).

[27] L. Cuchet, B. Rodmacq, S. Auffret, R. C. Sousa, and B. Dieny, Applied Physics Letters 105, 052408 (2014). 